\title{
Compressed Sensing Algorithm for Real-Time Doppler Ultrasound Image Reconstruction
}

\author{
Sulieman Mohammed Salih Zobly \\ Department of Medical Physics \& Instrumentation, National Cancer Institute, University of Gezira, Wad Medani, Sudan
}

\section{Email address:}

sulieman16@yahoo.com

\section{To cite this article:}

Sulieman Mohammed Salih Zobly. Compressed Sensing Algorithm for Real-Time Doppler Ultrasound Image Reconstruction. Mathematical Modelling and Applications. Vol. 2, No. 6, 2017, pp. 75-80. doi: 10.11648/j.mma.20170206.14

Received: January 31, 2017; Accepted: March 6, 2017; Published: December 18, 2017

\begin{abstract}
A Doppler ultrasound signal has been reconstructed using different compressed sensing algorithms. With compressed sensing it's possible to reconstruct signals and images using a few numbers of measurements so as to overcome the limitation of sampling in a real-time Doppler ultrasound sonogram. In this work we want to compare different compressed sensing algorithms used for Doppler ultrasound signal reconstruction so as to select the best algorithm that, gives a real-time Doppler ultrasound image and maintain quality. The result shows that regularized orthogonal matching pursuit reconstruction algorithm reconstructs the Doppler signal and gives Doppler spectrum in a real-time with high quality also $\ell 1$-norm reconstructs the Doppler signal and gives Doppler spectrum with a good quality, but the reconstruction time was very long.
\end{abstract}

Keywords: Doppler Ultrasound Signal, Compressed Sensing, Signal Reconstruction, $\ell 1$-Norm, Regularized Orthogonal Matching Pursuit

\section{Introduction}

Doppler ultrasound is one of the most important non-invasive techniques for measuring and monitoring blood flow within the body. Doppler instruments generate either continuous wave Doppler signal $(\mathrm{CW})$ or pulsed wave Doppler signal (PW). During the acquisition of Doppler data a train of pulses transmitted repeatedly to be acquired from selected region of interest. In most cases of Doppler signal acquisition done in more than one mode (duplex or triplex mode), this leads to reduction in frame rates for other modes, this reduction limit the ability to follow events in real-time. Also, rapid transmitting of ultrasound pulses to the same location increase the average power per unit area.

Nowadays different methods used to get a pictorial record of a Doppler shift signal of which the best and most commonly used is real-time spectral analysis. The output of the spectral analyzer is usually represented as spectrograms which show the Doppler spectrum as an intensity modulated line at a given time in real-time. This allows the sonographer to check the time-varying velocity from the output of this spectrogram.

Sparse signal can be approximately reconstructed efficiently from a small number of non-adaptive linear measurements. This process is known as compressed sensing (CS) [1]. In CS a few numbers of measurements of the signal samples will be considered to reconstruct the signal. This signal can be reconstructed with a good accuracy from much fewer measurements by a non-linear procedure. CS has been applied to reconstruct different medical imaging systems and many articles have been published in this area such as its application in Magnetic resonance imaging (MRI) [2], computed tomography (CT) [3], electroencephalogram (EEG) [4]. Beside those applications, CS has been applied to reconstruct Doppler ultrasound signal using different reconstruction algorithms to overcome the limitation of sampling [5 - 8].

In this work we want to compare between different compressed sensing reconstruction algorithms used for Doppler ultrasound signal reconstruction to select the best algorithm that gives lower reconstruction time and real time Doppler ultrasound image without affecting the image quality.

\section{Compressed Sensing}

Compressed sensing is a signal processing technique for efficiently reconstructing signals. CS started in 2006 when Donaho published his first work indicating that it's possible to 
reconstruct signals and images from a few numbers of measurements [1]. To work with a low number of measurements, compressed sensing theory requires the sensed signal to be sparse in a given orthogonal basis and the sensing vectors to be incoherent with this basis.

The main problem in CS is to recover sparse signal $\mathrm{x}$ from a set of measurements $y$, assume that $y$ is a linear measurement given by:

$$
\boldsymbol{y}=\boldsymbol{A X}
$$

Where $\mathrm{x}$ is $\mathrm{N}-\mathrm{by}-1, \mathrm{y}$ is M-by-1 vector containing the compressive measurements and $\mathrm{A}$ is $\mathrm{M}-\mathrm{by}-\mathrm{N}$ measurement matrix. The most difficult part of signal reconstruction is to identify the location of the largest component in the target signal. The signal reconstruction algorithm must take the $\mathrm{M}$ measurement in the vector $y$, where $N>>M$. Several algorithms for recovering sparse $\mathrm{x}$ from a limited number of measurements have been proposed such as $\ell 1$-norm and orthogonal matching pursuit.

Different CS algorithms have been used for Doppler ultrasound signal reconstruction and successfully reconstruct the signal with high performance. The algorithms used for Doppler signal reconstruction are $\ell 1$-minimization [9], orthogonal matching pursuit (OMP) [10], compressive sampling matching pursuit (CoSaMP) [11], regularized orthogonal matching pursuit (ROMP) [12] and multiple measurement vectors [13]. All the reconstruction algorithms used to reconstruct Doppler ultrasound signal exactly using a few numbers of measurements with a variation in reconstruction time and the quality of the reconstructed image.

\section{Methods}

To acquire Doppler ultrasound signal many samples required so as to calculate the sonogram. Doppler ultrasound signal was sampled non-uniformly in a random fashion and then reconstructed using five different CS reconstruction algorithms to generate the Doppler ultrasound spectrogram from a few numbers of measurements. The measurement model was as follows:

$$
f=A X
$$

Where $\mathrm{f}$ is $\mathrm{M}-\mathrm{by}-1$ vector containing the measurements, A is $\mathrm{M}-\mathrm{by}-\mathrm{N}$ measurement matrix and $\mathrm{X}$ is $\mathrm{N}-\mathrm{by}-1$ vector. The measurement vector $M$ used in this case was a Doppler signal with a length of 2032 point. This measurement gives sparsity properties on the other basis, which is solved by using one of the reconstruction algorithms. Figure 1 shows the extraction of Doppler signal, sampling and application of CS algorithms to the signal for Doppler spectrum reconstruction.

\subsection{1-Minimization}

1 -minimization is one of the most important compressed sensing algorithms used and applied in the signal processing field in the last few years. This algorithm uses an accelerated convergence to give the optimal rate for this class of problem and smoothing technique that replace the non-smooth $\ell 1$ norm with a smooth version [14]. If the measurement model expressed as follows:

$$
f=A X
$$

Where $\mathrm{f}$ is the $\mathrm{M}$ by 1 vector and $\mathrm{A}$ is the $\mathrm{M}$ by $\mathrm{N}$ matrix

This model can be solved by using $\ell 1$ minimization algorithm expressed as follows:

$$
\min \|\boldsymbol{x}\|_{\ell 1} \text { s. t. }\|\boldsymbol{A} \boldsymbol{x}-\boldsymbol{f}\|_{\ell 1} \leq \boldsymbol{\varepsilon}
$$

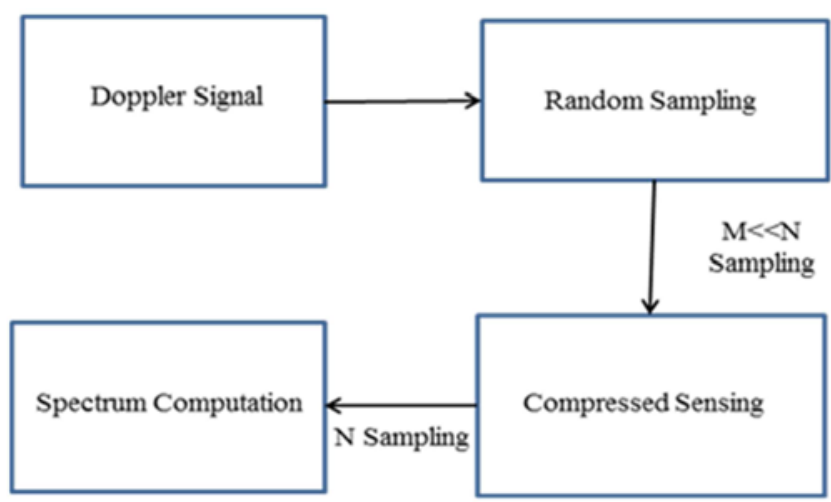

Figure 1. The steps of Doppler signal reconstruction via compressed sensing algorithms.

\subsection{Orthogonal Matching Pursuit (OMP)}

OMP is a greedy algorithm proposed for sparse recovery without knowing the error vector beside its simplicity and fastness for high dimension sparse signal recovery [15]. This algorithm can be easily implemented in practice and recover signal with high probability. The algorithm begins by finding the column of a mostly related with the measurements, then repeated this step by correlating the columns with the signal residual, which is obtained by subtracting the contribution of a partial estimate of the signal from the original measurement vector.

\subsection{Compressive Sampling Matching Pursuit (CoSaMP)}

CoSaMP is an iterative recovery algorithm used to recover signals using measurement matrices [11]. The concept of this algorithm is same as OMP algorithm, but, does a limited search at each step, it adds more than one coordinate at a time and then it discards the least useful coordinates.

If we consider a measurement matrix A is M-by-N satisfy the restricted isometry property, the sample vector can be expressed as follows:

$$
y=A x+e
$$

Where e is the error

The algorithm can produce a k-sparse approximation, which satisfy 


$$
\|\boldsymbol{x}-\mu\|_{2} \leq \boldsymbol{C} \cdot \max \left\{\eta, \frac{1}{\boldsymbol{k}}\|\boldsymbol{x}-\boldsymbol{x} \boldsymbol{k} / 2\|_{1}+\|2\|_{2}\right\}
$$

\subsection{Regularized Orthogonal Matching Pursuit (ROMP)}

Regularized orthogonal matching pursuit is a greedy algorithm used to reconstruct sparse signal. If we consider the equation (3), the signal $\mathrm{x}$ with a few non-zero coefficient can be solved by using a few numbers of measurements [16]. The difference between this algorithm and OMP is instead of choosing only one biggest correlation at each iteration, we choose biggest absolute coefficients. By this the signal can be recovered perfectly without going through all iterations

\subsection{Multiple Measurement Vector (MMV)}

MMV is a model used to find the column most related to the measurements and assume that signals matrices have same sparse structure. Several computation algorithms have been proposed to solve the MMV problem. The OMP is the most suitable algorithm that can find the sparsest solution for MMV [17].

The reconstruction algorithms were evaluated using three different performance measures, peak signal to noise ratio (PSNR), root mean square error (RMSE) and elapse time.

Root mean square error is one of the most important performance measure used to evaluate the image performance. The RMSE calculated for two images I and II with dimension of (m-by-n), where I is the original image and II is the reconstructed image. The RMSE measurement is easily computed by the square root of (mean square error MSE) the average squared difference between every pixel in recovering image and the original image. The RMSE calculated as follows:

$$
\begin{gathered}
M S E=\frac{1}{n m} \sum_{i=0}^{m-1} \sum_{j=0}^{n-1}[I(i, j)-I I(i, j)]^{2} \\
R M S E=\sqrt{M S E}
\end{gathered}
$$

Where, I(i,j) and II $(i, j)$ are the pixel values of the original and recovered image respecti vely and $m, n$ are the size of an image.

PSNR reflects the differences of the information contained between an original and recovered image. The PSNR numbers are reported in Decibels $(\mathrm{dB})$ as a measure of the relative weight between two images. A higher number in $\mathrm{dB}$ indicates a higher correlation. The PSNR is directly proportional to the image quality. When PSNR is higher this indicates that the reconstruction is of higher quality. PSNR calculated as follows:

$$
\boldsymbol{P S N R}=20 \log _{10}(255 / \boldsymbol{R M S E}) \boldsymbol{d B}
$$

The reconstruction time was calculated for each algorithm using different numbers of points several times and the average was calculated.

\subsection{Experimental Verification}

Array of ultrasound data with a length of 2032 point was collected from experimental Doppler ultrasound systems to reconstruct Doppler ultrasound spectrum. Software written in Matlab (Mathworks, MA) was developed to generate Doppler spectrum beside reconstructed Doppler spectrum. The Doppler signal undersampled randomly to reduce the length of the signal to other different lengths (here, five different lengths were used), $20 \%, 40 \%, 60 \% 80 \%$ and $100 \%$ of the data. Five compressed sensing algorithms were developed in Matlab using non-linear conjugate gradient methods ( $\ell 1$-minimization, orthogonal matching pursuit (OMP), compressive sampling matching pursuit (CoSaMP), regularized orthogonal matching pursuit (ROMP) and multiple measurement vectors) were used to reconstruct the Doppler signal exactly. The reconstructed signals were used to generate the Doppler spectrum. The reconstructed image quality evaluated using three different performance measures PSNR, RMSE and entropy. However, the image judged by an expert sonographer to verify there is no missing features in the reconstructed image.

\subsection{Doppler Signal Reconstruction}

Doppler signal with a length of 2032 was downloaded from H. Torp group, software written in Matlab was developed to generate both original and reconstructed Doppler spectrum. The Doppler signal was undersampled randomly and reconstructed by using five different compressed sensing reconstruction algorithms. Five different numbers of measurements were used to perform the reconstruction. The reconstruction algorithm software also was developed in Matlab.

\section{Result and Discussion}

The reconstruction was performed perfectly using different reconstruction algorithms and different numbers of measurements by sampling the signal randomly. The resulting signal reconstructed as follows:

Under sampled signal was reconstructed firstly with

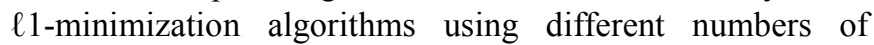
measurements. The signal was used then to generate the Doppler spectrum using a program developed in Matlab. Figure 2 shows the reconstructed spectrum using different numbers of measurements. The result shows that the algorithm reconstruct the Doppler spectrum perfectly even with a fewer number of points. However, the image quality decreased as the amount of points decreased, but a higher number of points increase the computation and the reconstruction time. 

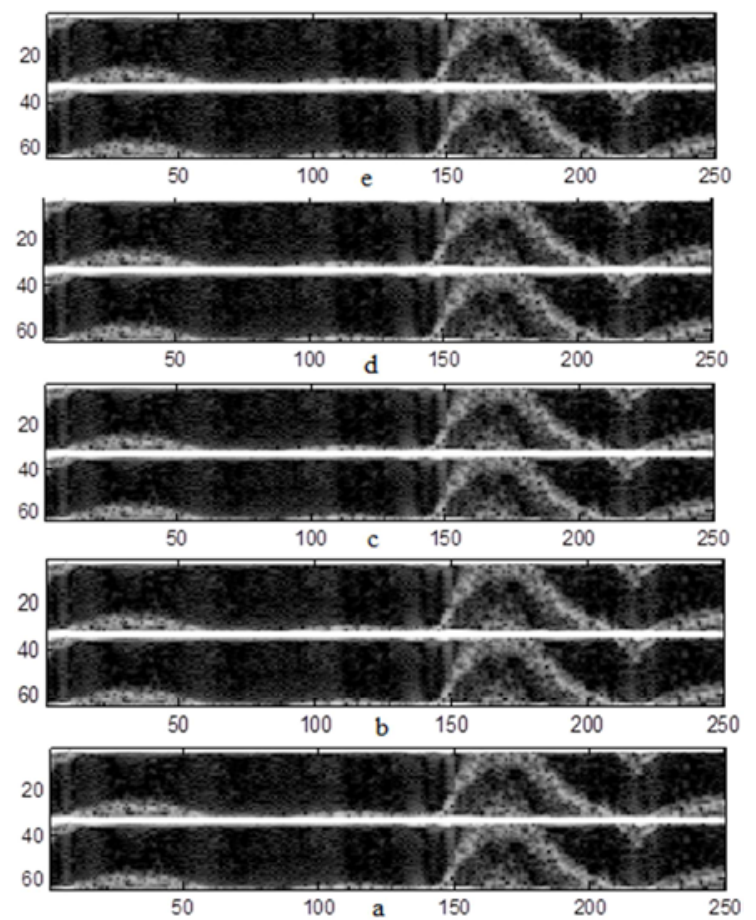

Figure 2. The reconstructed signal with $\ell_{1}$-norm using different numbers of points (a) $5 \%$ point (b) $20 \%$ point (c) $40 \%$ point (d) $60 \%$ point (e) $80 \%$ point.

Figure 3 shows the Doppler spectrum reconstructed via OMP algorithm using different numbers of measurements. The result shows that the algorithm reconstructs the Doppler signal and generates the Doppler spectrum, even by using fewer numbers of points. The quality of the images was evaluated by using three different performance measures.
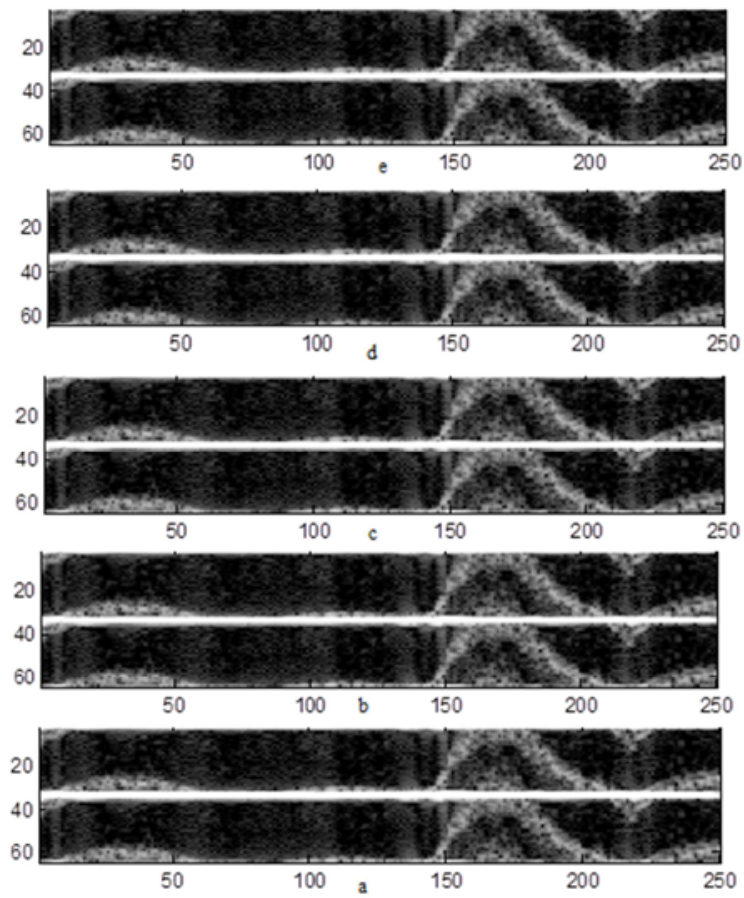

Figure 3. The reconstructed signal with OMP using different numbers of points (a) $5 \%$ point (b) $20 \%$ point (c) $40 \%$ point (d) $60 \%$ point (e) $80 \%$ point.

Figure 4 shows the reconstructed Doppler spectrum using a
CoSaMP algorithm with different numbers of points. Doppler spectrum reconstructed perfectly via COSAMP CS algorithm even when fewer numbers of points were used.
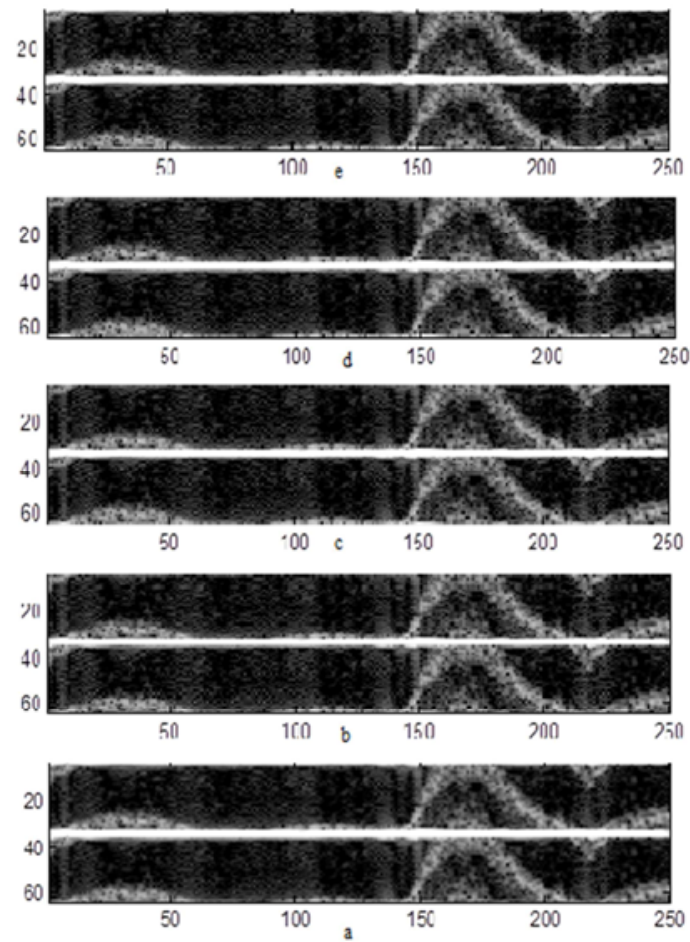

Figure 4. The reconstructed signal with CoSaMP using different numbers of points (a) $5 \%$ point (b) $20 \%$ point (c) $40 \%$ point (d) $60 \%$ point (e) $80 \%$ point.

Figure 5 shows the Doppler spectrum reconstructed via ROMP algorithm using different numbers of points. The algorithm reconstructs the spectrum perfectly with high and a few numbers of measurements.
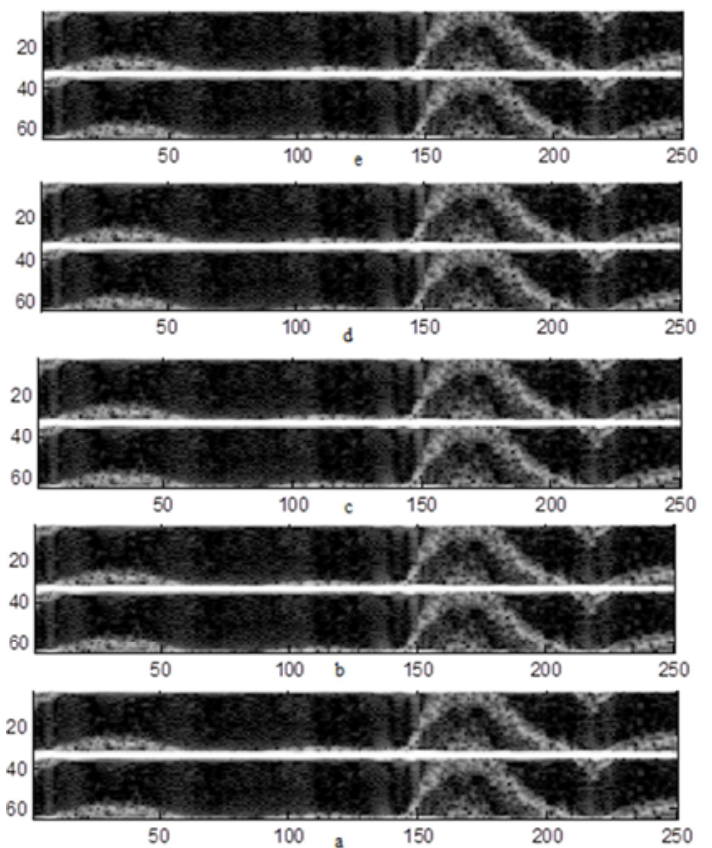

Figure 5. The reconstructed signal with ROMP using different numbers of points (a) $5 \%$ point (b) $20 \%$ point (c) $40 \%$ point (d) $60 \%$ point (e) $80 \%$ point. 
Figure 6 shows the recovered spectrum using different numbers of measurements via MMV algorithm. The result shows that the spectrum reconstructed perfectly using different numbers of points.
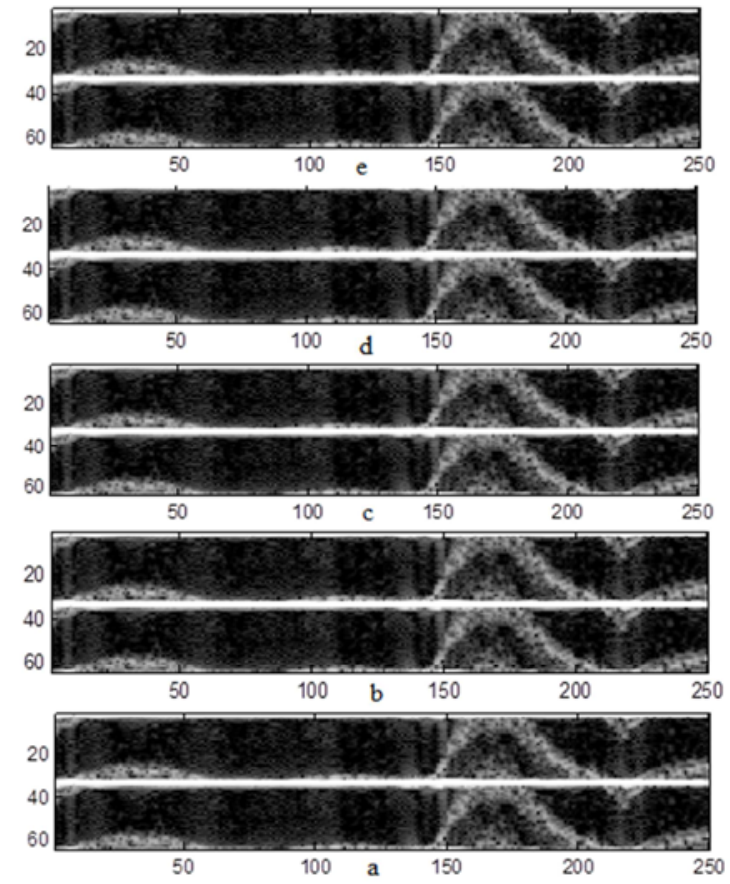

Figure 6. The reconstructed signal with $M M V$ using different numbers of points (a) $5 \%$ point (b) $20 \%$ point (c) $40 \%$ point (d) $60 \%$ point (e) $80 \%$ point.

The reconstruction time for each algorithm was calculated using different numbers of points several times and the average was calculated. Figure 7 shows the reconstruction time for the five algorithms used in this work. The result shows that ROMP gives the lowest reconstruction time among all the reconstruction algorithms used ( 0.02 second), this algorithm can be used to give Doppler images in real time. $\ell 1$ norm gives the highest reconstruction time ( 9 second) this algorithm can be used to reconstruct the Doppler signals, but can't give a real time Doppler image because of its long reconstruction time. OMP reconstructs the signal with reasonable time, but higher than ROMP algorithm. Others algorithms reconstruct the signal perfectly, but with interval time higher than ROMP and lower than the $\ell 1$ - norm.

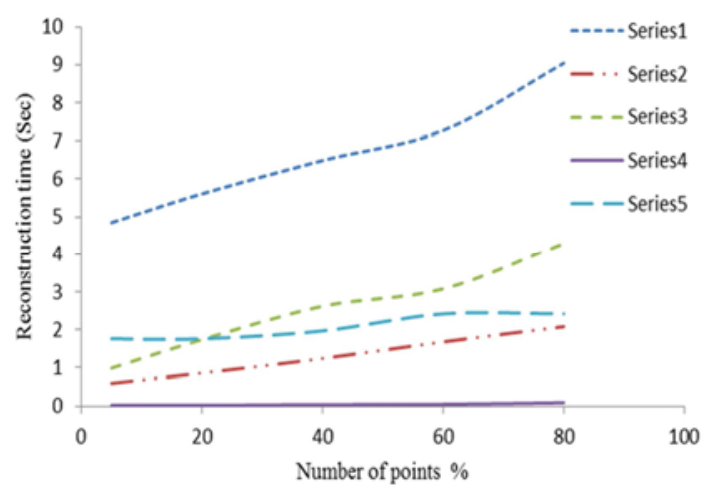

Figure 7. Compression of reconstruction time and number of point for different algorithms.
The RMSE was calculated from all images reconstructed using CS algorithms, the result shown in figure 8 . The result shows that the error decreased as the number of points increased. MMV and $\ell 1$-norm algorithm gives the highest RMSE among all the algorithms used and ROMP and OMP algorithms give lower RMSE. Comparing RMSE calculated from the algorithms, ROMP algorithm gives lower RMSE.

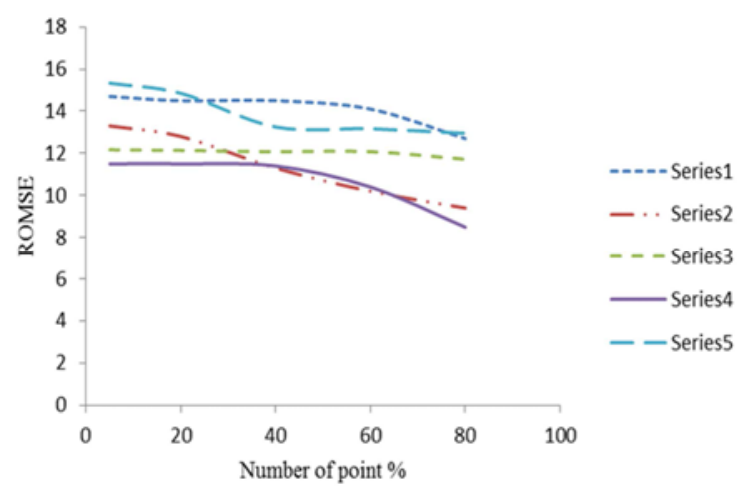

Figure 8. Compression of RMSE and number of point for different algorithms.

PSNR is the most important performance measure form image quality evaluation. The quality of image reconstructed with CS algorithm was shown in figure 9. The result shows that ROMP gives higher PSNR (best reconstructed images). MMV algorithms give lower PNSR (worst reconstructed image). The quality of image depends on the number of points used for the reconstruction. The quality of image increased as the number of measurement used for reconstruction increased.

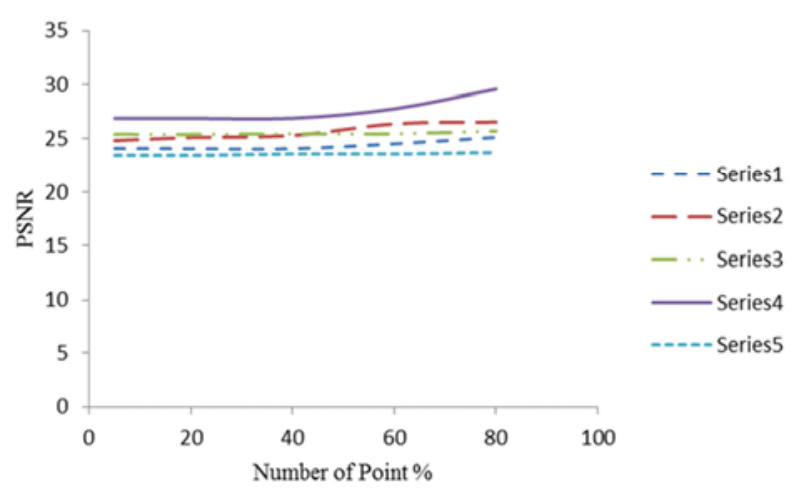

Figure 9. Compression of PSNR and number of point for different algorithms.

\section{Conclusion}

Doppler ultrasound has been reconstructed perfectly by using different compressed sensing algorithm and different numbers of measurement. The reconstruction performed using the program developed in MATLAB to generate the Doppler spectrum beside the reconstructed Doppler spectrum image. The result shows that the CS algorithms used can reconstruct the Doppler spectrum by using a few numbers of points with a good quality. The quality of reconstructed spectrum depends on the number of points used for reconstruction, the quality of images degraded as the number of measurements decreased. From compression the quality of images reconstructed with 
ROMP was the best. The MMV algorithm gives the worst reconstructed images among the algorithms used in this work. The reconstruction time from all the algorithms was calculated, ROMP reconstructs image in 0.02 second and this gives the real Doppler image. Higher reconstruction time was from $\ell 1$-norm algorithm was 9 second. Beside the recovery time and the quality the error was calculated for each image, the result shows that ROMP gives lower error and $\ell 1$-norm gives higher error. We can conclude that ROMP was the best algorithm can reconstruct Doppler images with very low error, high quality and gives Doppler images in a real time.

\section{References}

[1] D. Donoho, "Compressed Sensing", IEEE Trans. Info. Theory, vol. 52 (4), pp. 1289 - 1306, 2006.

[2] M. Lusting, D. Donoho, J. Pauly, Sparse MR: The Application of Compressed Sensing For Rapid MR Imaging, Magnetic Resonance in Medicine, 58 (6), pp. 1182 - 1182, 2007.

[3] H. Yu, G. Wang, Compressed Sensing Based Interior Tomography, Physics in Medicine and Biology, 54, 2791 $2805,2009$.

[4] S. Aviyente, Compressed Sensing Framework for EEG Compression, IEEE Statistical Signal Processing / SP 14th Workshop, pp. 181 - 184, 2007.

[5] S. Zobly and Y. Kadah, "Compressed Sensing: Doppler Ultrasound Signal Recovery by Using Non-uniform Sampling \& Random Sampling", Proc IEEE, 2011.

[6] S. Zobly and Y. Kadah, "Novel Doppler Ultrasound Data Acquisition Framework Based on Compressed Sensing", International Conference on: Advances in Biomedical Engineering, pp. 137 - 138, 2011.

[7] S. Zobly and Y. Kadah, "Orthogonal Matching Pursuit \& Compressive Sampling Matching Pursuit for Doppler Ultrasound Signal Reconstruction”, Proc IEEE, 2012.
[8] S. Zobly and Y. Kadah, "Multiple Measurements Vectors Compressed Sensing for Doppler Ultrasound Signal Reconstruction", International Conference in Computing, Electronics and Electrical Engineering, Proc IEEE, 2013.

[9] E. Candes, J. Remborg and T. Tao, "Robust uncertainty principles: Exact signal reconstruction from highly incomplete frequency information,” IEEE Trans. Info. Theory, vol. 52 (2), pp. $489-509,2006$.

[10] J. Tropp and A. Gilbert, "Signal recovery from random measurements via orthogonal matching pursuit", IEEE Trans. Info. Theory, vol. 53 (12), 2007.

[11] D. Needell, J. Tropp, COSAMP: Iterative Signal Recovery from Incomplete and Inaccurate sampling, ACM Technical Report 2008-01, California Institute of Technology, 2008.

[12] D. Needell, R. Vershynin, Signal Recovery from Incomplete and Inaccurate Measurements via Regularized Orthogonal Matching Pursuit, IEEE J. Select. Top. Signal Processing, 4 (2), pp. $310-316,2010$.

[13] I. Gorodnitsky and B. Rao, "Sparse Signal Reconstruction from Limited Data using FOCUSS: A re-weighted minimum norm Algorithm", IEEE Transaction on Signal Processing, vol. 45 (3), pp. $600-616,1997$.

[14] S. Beaker, J. Boben, E. Candes, A fast and Accurate First-order methods for sparse recovery. Available: https://www.eecs.berkeley.edu/ yang/paper/YangA_ICIP2010. pdf.

[15] D. Needell, J. Tropp, R. Vershynin, “Greedy Signal Recovery Review," 2008. Available: www.acm.caltech.edu/ jtropp/conf/NTV08-greedy-signal-asil omar.pdf.

[16] S. Zobly, Y. Kadah, Processing Methodologies for Doppler Ultrasound, PhD Thesis, 2012.

[17] H. Lu, X. Long and J. Lv, "A Fast Algorithm for Recovery of Jointly Sparse Vector Based on the Alternating Direction Methods", International Conference on Artificial Intelligence and Statistic, vol. 15, pp. 461- 469, 2011. 